\title{
BK Virus-Associated Urothelial Carcinoma in a Patient with Peripheral Blood Stem Cell Transplantation for Acute Lymphoblastic Leukemia: A Case Report
}

\author{
Juntaro Koyama ${ }^{a}$ Yoshihide Kawasaki $^{a}$ Shingo Kimura ${ }^{a}$ Takuma Sato $^{\text {a }}$ \\ Shuichi Shimada ${ }^{a}$ Naoki Kawamorita ${ }^{a}$ Shinichi Yamashita ${ }^{a}$ Ryo Nakagawab \\ Akihisa Kawajiri $^{b}$ Koichi Onodera $^{b}$ Yasushi Onishib ${ }^{b}$ Koji Mitsuzuka $^{a}$ \\ Mika Watanabec Akihiro Ito ${ }^{a}$ \\ aDepartment of Urology, Tohoku University Graduate School of Medicine, Sendai, Japan; \\ ${ }^{b}$ Department of Hematology and Rheumatology, Tohoku University Graduate School of \\ Medicine, Sendai, Japan; 'Division of Pathology, Tohoku University Hospital, Sendai, Japan
}

\section{Keywords}

BK virus - Bladder · Urothelial carcinoma - Hemorrhagic cystitis - Peripheral blood stem cell transplantation

\begin{abstract}
Bladder tamponade due to hemorrhagic cystitis caused by BK virus in immunocompetent patients is familiar to urologists. BK virus is an important cause of nephropathy and graft loss in kidney transplant recipients. Although urothelial carcinoma of the bladder in kidney transplant recipients with persistent BK viruria is known, BK virus-associated urothelial carcinoma (BKVUC) in peripheral blood stem cell transplantation recipients is not as well known. $A$ 54-year-old man with acute lymphoblastic leukemia was treated in the Department of Hematology of our hospital. After recurrence 25 months later, he received chemotherapy for half a year and underwent peripheral blood stem cell transplantation. He achieved temporarily complete remission, but he developed hematuria with BK virus-positive result 1 month after peripheral blood stem cell transplantation. One month later, he developed bladder tamponadediagnosed hemorrhagic cystitis due to BK virus in our Urological Department. We performed transurethral coagulation to manage hemorrhage and removed a bleeding lesion in the bladder wall. Pathological examination of the removed bladder wall revealed pT1 stage BKVUC. We found that bladder tamponade could have led to reactivation of BK virus in this immunocompetent patient. This could be the first report of BKVUC of the bladder found in a peripheral blood stem cell transplantation recipient with close urological follow-up for 24 months. Adequate removal of bleeding lesions from the bladder mucosa with appropriate timing during hemorrhagic cystitis due to BKVUC could be essential to achieve good outcomes.
\end{abstract}




\section{Introduction}

BK virus was initially isolated from a renal transplant patient in 1971 and is part of the polyomavirus family [1]. Several reports have suggested that BK virus may play a significant role in the pathogenesis of bladder cancer [2]. Furthermore, significant associations between urine cytological evidence of polyomavirus infection and bladder cancer have been demonstrated in immunocompetent patients [3]. On the other hand, BK viruria and hemorrhagic cystitis are reportedly more frequent in allogeneic hematopoietic stem cell transplant patients receiving full conditioning and unrelated-donor HLA-mismatched grafts [4]. Details of the pathogenesis, prognosis, and treatment of BK virus-associated urothelial carcinoma (BKVUC) are not well understood.

\section{Case Report}

A 54-year-old man presented to the Department of Hematology of our hospital with a diagnosis of acute lymphoblastic leukemia. Other than leukemia, his medical history was only surgery for cholesteatoma. He denied any history of tobacco use. He achieved remission after receiving chemotherapy (vincristine + daunorubicin + cyclophosphamide + L-asparaginase), but recurrence was identified 25 months later. He received HLA haploidentical stem cell transplantation with fludarabine and busulfan for conditioning, and tacrolimus, posttransplant cyclophosphamide, mycophenolate mofetil for graft versus host disease prophylaxis, and complete remission had been confirmed. However, 1 month after peripheral blood stem cell transplantation, he developed macrohematuria and his urinary viral examination was positive for BK virus. One month later, bladder tamponade developed multiple times and hemorrhagic cystitis due to BK virus was diagnosed in our Urological Department. Blood test showed severe bone marrow suppression attributed to the effects of chemotherapy. Despite daily transfusions, platelet counts remained at 30,000-40,000/ $\mu \mathrm{L}$. Cystoscopy revealed a massive blood clot and bleeding from the right wall. We performed transurethral electrocoagulation under a diagnosis of hemorrhagic cystitis. A small nodule was found on the right wall during surgery, and so it was resected. Histological examination of hematoxylin-eosinstained tissue sections demonstrated high-grade urothelial carcinoma with submucosal invasion with hemorrhagic cystitis (Fig. 1). Immunohistochemical staining of the urinary bladder performed for distinction between reactive proliferative changes and malignant epithelia revealed positive results for p53, p16, SV40, and CK7 and negative results for CK20. Finally, we concluded with the pathological diagnosis of pT1 stage BKVUC. Preoperative urinary cytology was negative for polyomavirus-infected cells. Contrast-enhanced computed tomography revealed no metastasis of bladder cancer.

One month later, we performed second transurethral electrocoagulation to control bleeding by electrocoagulation and adequately removed the lesion soon after relapse of bladder tamponade. Pathological findings again revealed pT1 stage BKVUC. Our patient has been recurrence free from BKVUC for 24 months after transurethral controlling of hemorrhage cystitis and single bladder instillation therapy with anticancer agents.

\section{Discussion and Conclusion}

BK virus reactivation can occur under an immunosuppressed status, notably in patients after kidney transplantation or blood stem cell transplantation. According to several studies, primary BK virus infection occurs in $90 \%$ of the population by 10 years old, but almost all 


\section{Case Reports in Oncology}

\begin{tabular}{l|l}
\hline \multicolumn{2}{l}{ Case Rep Oncol 2021;14:8-12 } \\
\hline DOI: 10.1159/000511053 & $\begin{array}{l}\text { O 2021 The Author(s). Published by S. Karger AG, Basel } \\
\text { www.karger.com/cro }\end{array}$ \\
\hline
\end{tabular}
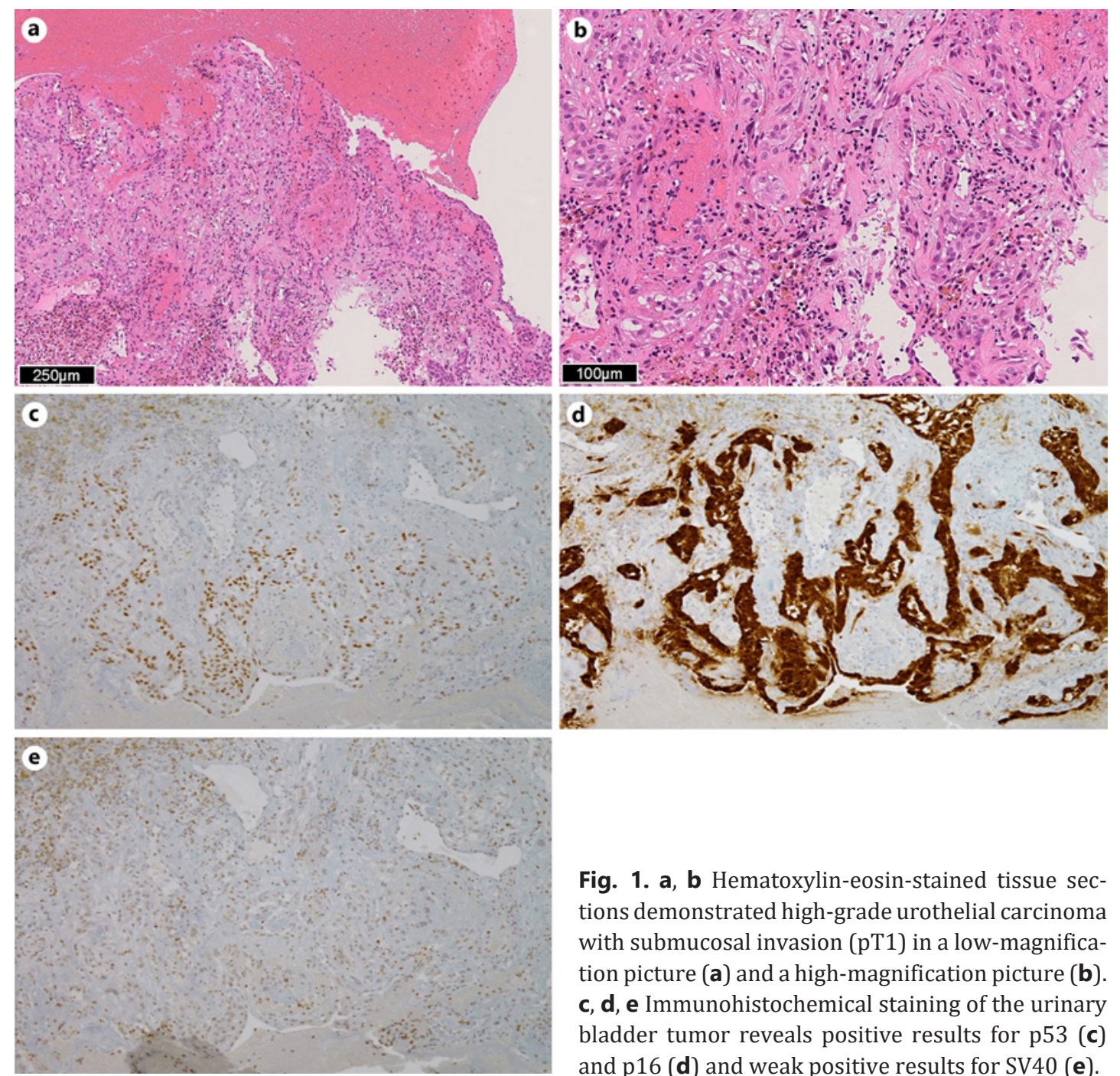

Fig. 1. a, b Hematoxylin-eosin-stained tissue sections demonstrated high-grade urothelial carcinoma with submucosal invasion (pT1) in a low-magnification picture (a) and a high-magnification picture (b). c, d, e Immunohistochemical staining of the urinary bladder tumor reveals positive results for p53 (c) and p16 (d) and weak positive results for SV40 (e).

people remain asymptomatic [5]. In our case, BK virus reactivation could have occurred immediately after transplantation, as hemorrhagic cystitis was found soon after peripheral blood stem cell transplantation. Cyclophosphamide had been administered for 3 years as an anticancer agent for leukemia and had probably contributed to reactivation of BK virus. BK virus is actually well known to be likely to provoke hemorrhagic cystitis in such immunocompromised individuals [6]. The possibility of a relationship between BK virus reactivation in immunosuppressed patients and oncogenesis in the urinary bladder was reported in 2013 [7]. In that report, BK virus showed potential for malignant transformation of activated cells, cell cycle shifts to proliferation and inhibition of apoptosis. BK virus could play significant roles in the pathogenesis of bladder cancer [8] and has been linked to urothelial carcinoma $[9,10]$.

BKVUC is likely to be found in an advanced stage and to show poor prognosis. It is supposed to coexist with BK virus-positive hemorrhagic cystitis. BKVUC in immunesuppressed patients with hemorrhagic cystitis is important to understand. We need to consider BKVUC hidden under hemorrhagic cystitis and achieve early diagnosis with histological examination to achieve good outcomes for patients. 
Diagnosis of BKVUC requires confirming the expression of SV40 T antigen. Expression of SV40 has been recognized as an important mediator to disable tumor suppressor genes (e.g., p53) [11]. SV40 is a protein coded by simian virus 40, which converts infected cells to malignant formation by denaturing proteins crucial for tumor suppression, such as p53 and pRB. Then, large $\mathrm{T}$ antigen binds to p53 after accumulation by stabilizing in BKVUC. Furthermore, p16 was reported to be strongly expressed and diffusely positive in invasive BKVUC [7]. Therefore, expression of p53, p16, and SV40 on histological examination were essential to diagnose BKVUC.

Excessive immunosuppression with calcineurin inhibitors, mycophenolate derivatives, high-dose steroids plus monoclonal antibodies could reportedly increase the chance of BK virus reactivation [12]. In our case, calcineurin inhibitors and mycophenolate derivatives were used for half a year before transplantation, and BK virus reactivation was thought to have occurred about 1 month after transplantation. When hemorrhagic cystitis develops in immunosuppressed patients, we need to consider BK virus reactivation causing UC as soon as possible for early diagnosis.

BKVUC of the bladder in kidney transplant recipients with persistent BK viruria has already been reported $[9,10]$. However, BKVUC in peripheral blood stem cell transplantation recipients is not well known. Innate immunity usually recovers during the several months after peripheral blood stem cell transplantation, and the reconstitution of adaptive immunity occurs over the first 1-2 years [13]. BKVUC might be rare in patients with peripheral blood stem cell transplants because of the short duration of their immunocompromised condition. In contrast, our case has received chemotherapy with lymphocytic toxicity for a few years before peripheral blood stem cell transplantation. Therefore, he could be more likely to contract BKVUC of the bladder than others who underwent peripheral blood stem cell transplantation. Our report could be the first report of BKVUC of the bladder found in a patient with peripheral blood stem cell transplantation. BKVUC of the bladder is often found as an advanced cancer with muscle invasive tumor that needs to undergo total cystectomy. To date, a small number of reports have described a micropapillary variant with BKVUC. Hill et al. [14] reported that strong p53 positivity in micropapillary tumors suggested a molecular pathway of oncogenesis in a setting of BK virus infection. BK viruria may be a risk factor for this aggressive form of bladder cancer $[9,10]$. However, the relationship between BK virus and development of the micropapillary variant was not elucidated in those reports.

Our case underwent transurethral resection of bladder tumor (TURBT) and immediate intravesical instillation of mitomycin C (MMC). Because his pathological stage was T1, further intravesical instillation therapy with bacille Calmette-Guérin (BCG) could be an option. Intravesical BCG is familiar to urologists as safe and effective for administration even in immunologically compromised patients with bladder cancer [15]. However, he did not undergo intravesical BCG therapy because he needed further therapy for leukemia and did not show any evidence of recurrence of urothelial carcinoma for 24 months of close urological follow-up with cystoscopy and urine cytology. Since the immunosuppressive state continues in our case, he has still been at risk of bladder cancer relapse due to BK virus reactivation. We need to continue close follow-up with cystoscopy, urine cytology, and TURBT, as needed.

In conclusion, this could be the first report of BKVUC found in a patient with peripheral blood stem cell transplantation. BKVUC of the bladder is supposed to coexist with BK viruspositive hemorrhagic cystitis and is likely to be an advanced cancer with poor prognosis. We need to identify patients with hemorrhagic cystitis at risk of BKVUC treated with immunosuppressive therapy. Our report highlights that adequate removal of bleeding lesions from the bladder mucosa during hemorrhagic cystitis could contribute to good outcomes of BKVUC of the balder.

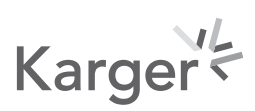




\section{Case Reports in Oncology}

Case Rep Oncol 2021;14:8-12

\section{Statement of Ethics}

The patient has given his written informed consent to the publication of his case including images.

\section{Conflict of Interest Statement}

The authors declare no conflict of interest in association with this article.

\section{Funding Sources}

There were no funding sources.

\section{Author Contributions}

All individuals who qualify as authors have been listed; each has participated in the conception and design of this work, the writing of the document, and the approval of the submission of this version.

\section{References}

1 Gardner SD, Field AM, Coleman DV, Hulme B. New human papovavirus (B.K.) isolated from urine after renal transplantation. Lancet. 1971 Jun 19;1(7712):1253-7.

2 Monini P, Rotola A, Di Luca D, De Lellis L, Chiari E, Corallini A, et al. DNA rearrangements impairing BK virus productive infection in urinary tract tumors. Virology. 1995 Dec 1;214(1):273-9.

3 Weinreb DB, Desman GT, Amolat-Apiado MJ, Burstein DE, Godbold JH Jr., Johnson EM. Polyoma virus infection is a prominent risk factor for bladder carcinoma in immunocompetent individuals. Diagn Cytopathol. 2006 Mar;34(3):201-3.

4 Giraud G, Priftakis P, Bogdanovic G, Remberger M, Dubrulle M, Hau A, et al. BK-viruria and haemorrhagic cystitis are more frequent in allogeneic haematopoietic stem cell transplant patients receiving full conditioning and unrelated-HLA-mismatched grafts. Bone Marrow Transplant. 2008 Apr;41(8):737-42.

5 Knowles WA. Discovery and epidemiology of the human polyomaviruses BK virus (BKV) and JC virus (JCV). Adv Exp Med Biol. 2006;577:19-45.

6 Rorije NM, Shea MM, Satyanarayana G, Hammond SP, Ho VT, Baden LR, et al. BK virus disease after allogeneic stem cell transplantation: a cohort analysis. Biol Blood Marrow Transplant. 2014 Apr;20(4):564-70.

7 Alexiev BA, Randhawa P, Vazquez Martul E, Zeng G, Luo C, Ramos E, et al. BK virus-associated urinary bladder carcinoma in transplant recipients: report of 2 cases, review of the literature, and proposed pathogenetic model. Hum Pathol. 2013 May;44(5):908-17.

8 Rollison DE, Sexton WJ, Rodriguez AR, Kang LC, Daniel R, Shah KV. Lack of BK virus DNA sequences in most transitional-cell carcinomas of the bladder. Int J Cancer. 2007 Mar 15;120(6):1248-51.

9 Alexiev BA, Papadimitriou JC, Chai TC, Ramos E, Staats PN, Drachenberg CB. Polyomavirus (BK)-associated pleomorphic giant cell carcinoma of the urinary bladder: a case report. Pathol Res Pract. 2013 Apr;209(4):255-9.

10 Bialasiewicz S, Cho Y, Rockett R, Preston J, Wood S, Fleming S, et al. Association of micropapillary urothelial carcinoma of the bladder and BK viruria in kidney transplant recipients. Transpl Infect Dis. 2013 Jun;15(3):283-9.

11 Zhou H, Huang HY, Shapiro E, Lepor H, Huang WC, Mohammadi M, et al. Urothelial tumor initiation requires deregulation of multiple signaling pathways: implications in target-based therapies. Carcinogenesis. 2012 Apr;33(4):770-80.

12 Wang HH, Liu KL, Chu SH, Tian YC, Lai PC, Chiang YJ. BK virus infection in association with posttransplant urothelial carcinoma. Transplant Proc. 2009 Jan-Feb;41(1):165-6.

13 Seggewiss R, Einsele H. Immune reconstitution after allogeneic transplantation and expanding options for immunomodulation: an update. Blood. 2010 May 13;115(19):3861-8.

14 Hill P, Slavin J, Goodman D. High-grade urothelial carcinoma in a kidney transplant recipient with BK virus infection. NDT Plus. 2009 Jun;2(3):246-9.

15 Herr HW, Dalbagni G. Intravesical bacille Calmette-Guérin (BCG) in immunologically compromised patients with bladder cancer. BJU Int. 2013 May;111(6):984-7. 